\title{
Design of Dynamic Controllers for Continuous Paths on Parallel Platforms (Slide Modes and PD+)
}

\author{
Angie Valencia ${ }^{1}$, Mauricio Mauledoux $^{1^{*}}$, and Claudia Castañeda ${ }^{1}$ \\ ${ }^{1}$ Militar Nueva Granada University, Mechatronics Engineering Program, Bogota Colombia
}

\begin{abstract}
A parallel robot is defined as a mechanism of closed kinematic chains with rigid movements, high speed, precision and better inertias, in respect to those with structures composed by open links. besides these configurations describe highly nonlinear behaviours, reflected in the complexity of their kinematic and kinetic models. Finally, these systems are integrated with control techniques that obtain the desired trajectories in this paper for continuous 3D printing applications. For this reason, the development of control techniques based on dynamics by torque control law using drivers for the actuators maintaining the desired torque are described, these control techniques allow to obtain continuous behaviours in the final effector in order to make uniform impressions for biopolymer scaffolds.
\end{abstract}

\section{Introduction}

Additive manufacturing is the basis of bio-printing systems for tissue engineering, in which an extruder head deposits material in successive layers, with movement guided by computational algorithms. In the development of pieces, it is important to correlate the geometric properties of the deposited material with the mechanical structure that will orient the printing system, because the inertia of the mechanism must be minimized in order to generate continuous paths reflected in the final effector [1,2].

There are mechanisms with links, joined one to one using linear or rotational actuators, for example there are XYZ Cartesian robots that combine independent linear movements in three-dimensional space, or serial manipulators that base their operation on the anatomy of human structure. Its main advantage lies in its large work space, and the number of configurations the final effector can reach, giving up the precision by the accumulated error in the joints, in addition to high control efforts by the sum of the moments of inertia generated by each element, which is reflected in actuators of higher torque and cost, compared to closed kinematic chains[3].

On the other hand, there are closed kinematic chains, characterized by having rigidity in high speed applications and accelerations of up to 50 times the value of gravity $[4,5,6]$. For its design, complex kinematic and dynamic calculations are required, due to non-linear behaviours, internal singularities, reduced workspace and non-acted joints [7, 8]. Among the most commonly used configurations are delta-type mechanisms consisting of three fixed motors located on a stationary base that move a terminal platform. The tripteron which is a cartesian parallel actuator capable of reaching linear configurations [9]. Finally, there is the hexa configuration with a structure like the delta-type, but it consists of six fixed motors that allow the position and orientation of the final effector [2].

The analysis of the structures is accompanied by the kinematic and dynamic models that linked to controllers allowing to obtain the desired behaviours on the final effector. As for the dynamic calculation, the implementation of the position, velocity and acceleration derivatives of the final effector is considered to know the value of the forces to be exerted to perform a movement or vice versa [10]. Then, control algorithms such as PIDs (proportional, integral and derivative) are designed that work with linear models [11], or techniques such as Sliding Modes that base their operation on the non-linear model, considering system uncertainties and external disturbances $[12,13]$.

Therefore, this document presents two control techniques for a Hexa-type platform; because it performs its movements according to the position of rotational actuators, which allows continuous behaviour in the final effector with respect to those whose transmission is carried out by mechanisms such as an endless screw, since these discretize the movements in function of the screw pitch. Finally, the requirements of additive manufacturing such as continuous printing and precision will be analysed, compared to the control efforts in order to select the technique that best suits the needs of the project.

\section{Robot Dynamics}

In Figure 1, the configuration of a Hexa-type robot is presented that allows to obtain desired behaviours in position $(x, y, z)$ and in orientation defined by Euler's angles $(\alpha, \beta, \gamma)$. For this, it is necessary to design control

Corresponding author: mauricio.mauledoux@unimilitar.edu.co 
techniques based on the dynamic model that considers the actuators' effort to generate movement.

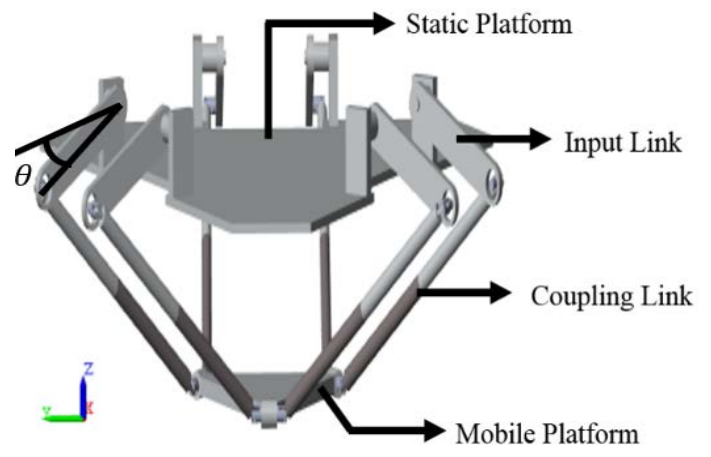

Fig. 1. Robot structure

In equation (1), it is present in a matrix form the positions, internal and external forces that determine the torque of the six generalized coordinates ( $\theta_{1}, \theta_{2}, \theta_{3}, \theta_{4}, \theta_{5}, \theta_{6}$ ), obtained according to the platform geometry and material, which define the lengths and weights of the links.

$$
M(q) \dot{q}+V(q, \dot{q})+G(q)+F_{v}=\tau
$$

Where $M$ denotes the mass or inertia matrix, $V$ the matrix of centrifugal and Coriolis forces, $G$ gravitational terms and $F_{v}$ represent the coefficients of viscous friction.

\section{Control Systems}

In this section, two types of control are shown, which are aimed at tracking the path based on the dynamics of the error.

\subsection{PD+}

This technique bases its control law on signals that are modified according to the weighting of the error and its derivative as shown in equation (2) and (3) and figure 2.

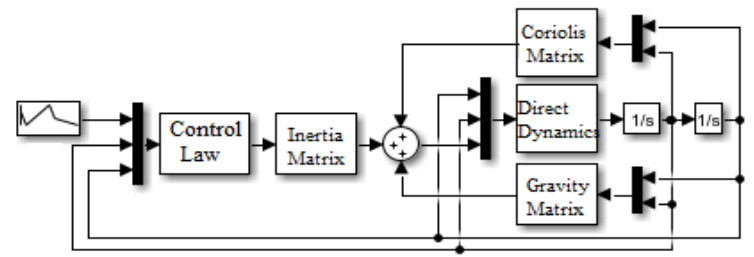

Fig 2 PD+ Control scheme

Additional centrifugal, Coriolis and gravitational forces are considered, as a parameter that modifies the force on the actuators.

$$
\begin{gathered}
k_{v}\left(\dot{q}_{d}-\dot{q}\right)+k_{p}\left(q_{d}-q\right)=\tau \\
M(q, \dot{q})\left(k_{v} \dot{e}+k_{p} e\right)+V(q, \dot{q})+G(q, \dot{q})=\tau
\end{gathered}
$$

\subsection{Sliding Modes (SM)}

It consists of guiding the trajectories of the system to a sliding surface in the phase diagram, which exists if in the vicinity the tangent or velocity vectors point in the direction of said surface as described in equation (4) and (5).

$$
\begin{gathered}
\sigma=q+k_{1} e+k_{2} \int e+k_{3} \iint e \\
\dot{\sigma}=k_{1} \dot{e}+k_{2} e+k_{3} \int e
\end{gathered}
$$

Then, the control is determined as the sum of the equivalent control $\left(\tau_{e q}\right)$ (equation (6)) and the attractive control $\left(\tau_{N}\right)$ (equation (7)), to obtain the control law described in equation (8).

$$
\begin{aligned}
& \tau_{e q}=G(q)+V(q, \dot{q}) \\
& +M(q)\left(k_{2} e+k_{1} \dot{e}+k_{3} \int e\right) \\
& \tau_{N}=M(q) L \cdot \operatorname{sgn}\left(q+k_{1} e+k_{2} \int e+k_{3} \iint e\right) \\
& \tau=G(q)+V(q, \dot{q}) \\
& +M(q)\left(k_{2} e+k_{1} \dot{e}+k_{3} \int e\right) \\
& +M(q) L \cdot \operatorname{sgn}\left(q+k_{1} e+k_{2} \int e+k_{3} \iint e\right)
\end{aligned}
$$

Equating equation (8) and robot dynamics, the error dynamics in equation (9) are obtained.

$$
\begin{gathered}
k_{2} e+k_{1} \dot{e}+k_{3} \int e+L \cdot \operatorname{sgn}\left(q+k_{1} e+k_{2} \int e+\right. \\
\left.k_{3} \iint e\right)=0
\end{gathered}
$$

\section{Analysis of results}

To corroborate the design of the controllers, the platform is subjected to a helical path in its final effector, from which the behavior of Figure 3 to 6 is obtained for the PD + control, with initial conditions 0 for $x, 0.01$ for $Y,-0.15$ for $Z, 0.1$ for $\alpha, \beta$ and $\gamma$

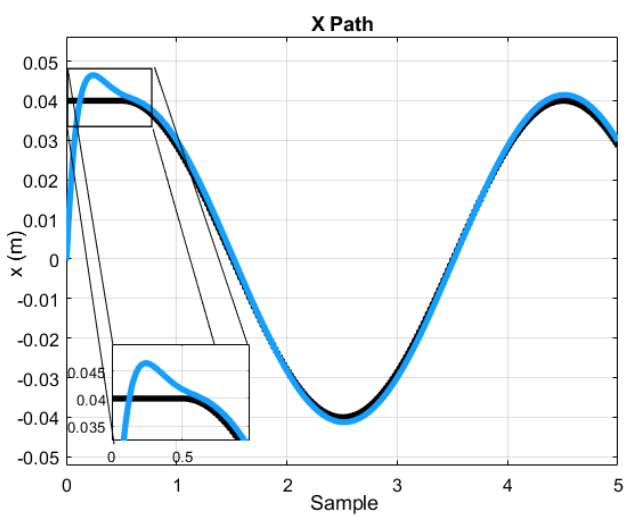

Fig $3 \mathrm{X}$-axis reference vs output $\mathrm{PD}+$ behavior 


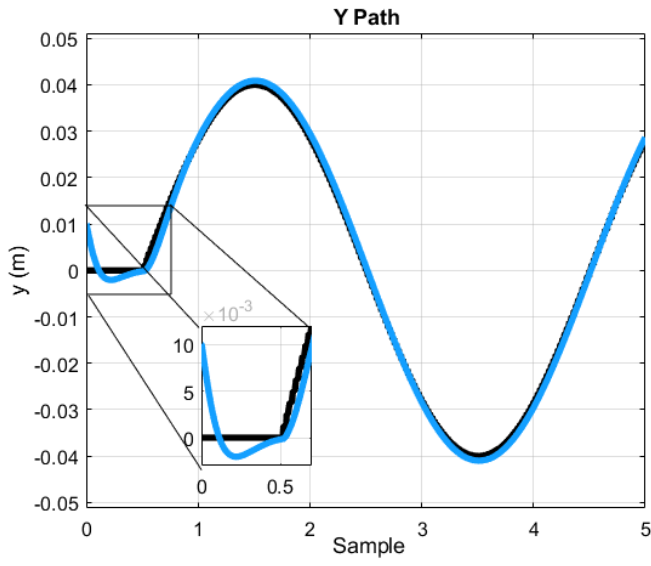

Fig $4 \mathrm{Y}$-axis reference vs output $\mathrm{PD}+$ behavior

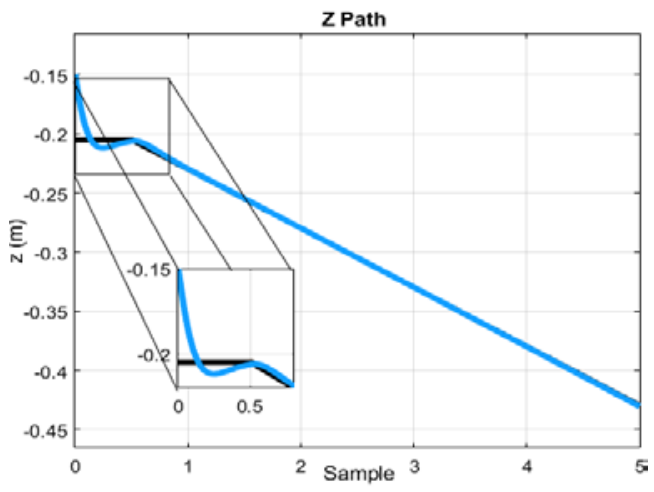

Fig 5 Z-axis reference vs output PD+ behavior

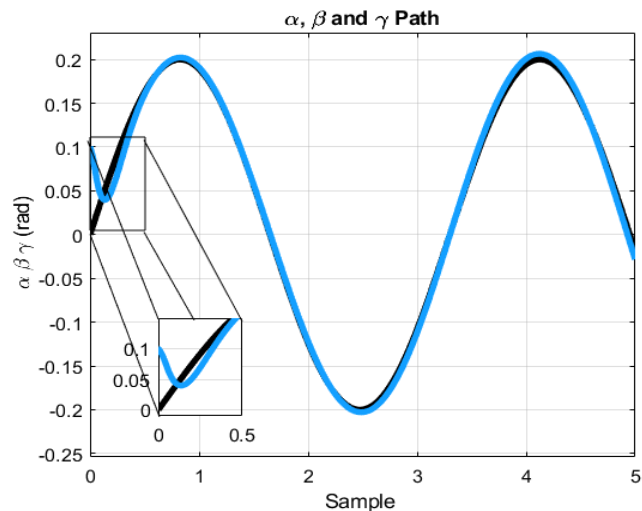

Fig 6 Orientation reference vs output PD+

Figure 7 to 10 , the behaviors of the platform with the controller are obtained by sliding modes, with initial conditions 0 for $x, 0.01$ for $Y,-0.15$ for $Z, 0.1$ for $\alpha, \beta$ and $\gamma$

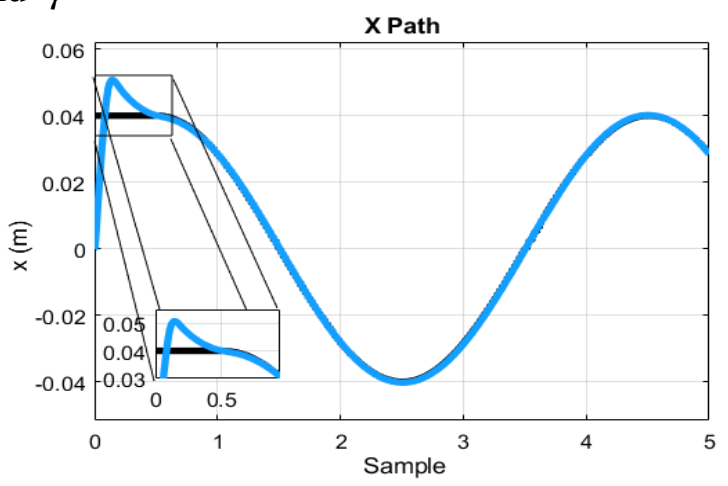

Fig $7 \mathrm{X}$-axis reference vs output Sliding Modes behavior

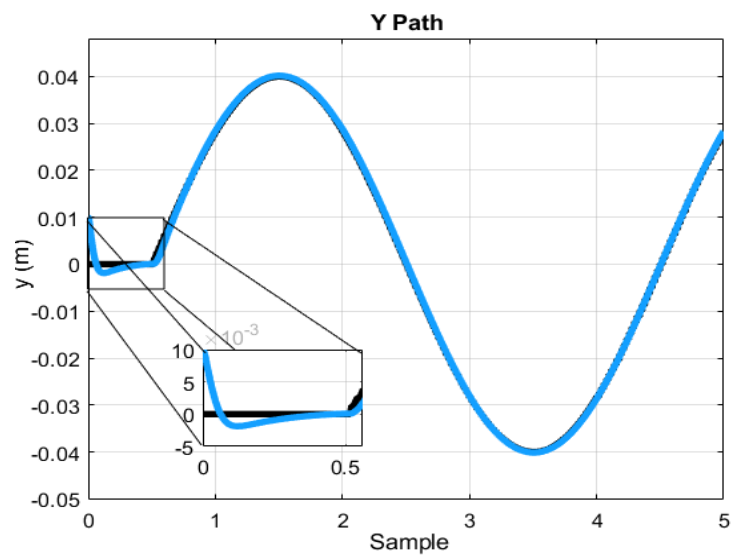

Fig 8 Y-axis reference vs output Sliding Modes behavior

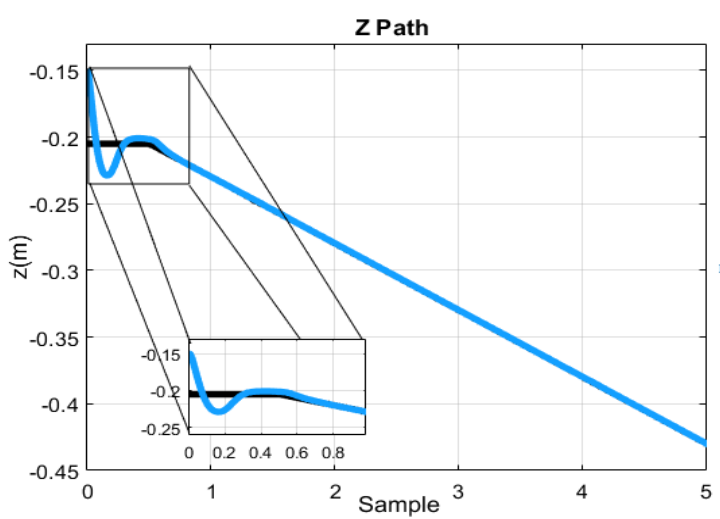

Fig $9 \mathrm{Z}$-axis reference vs output Sliding Modes behavior

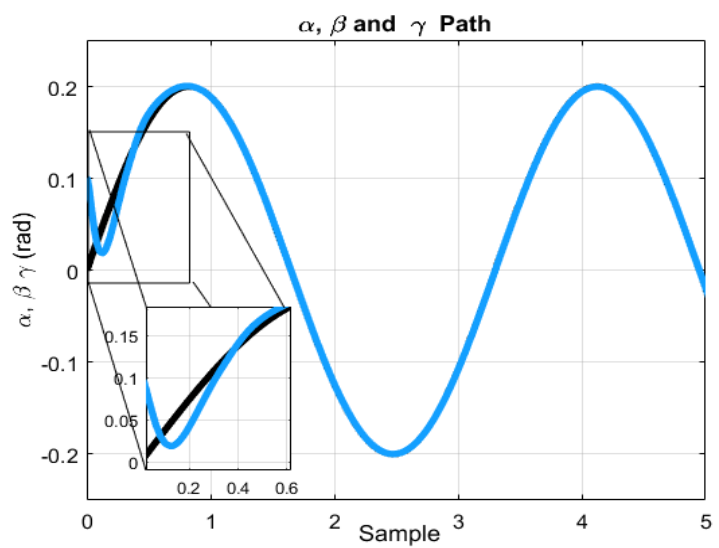

Fig 10 Orientation reference vs output Sliding Modes behavior From the above, the robustness of the control by SM is observed, and how this modified the robot behavior to get the desired trajectory in less than 0.4 seconds compared to the PD + that makes more than 0.5 seconds. Then Then the error present in the controls is observed in table 1, concluding that the control law by SM is better at tracking paths.

Table 1. Mean square error

\begin{tabular}{|c|c|c|}
\cline { 2 - 3 } \multicolumn{1}{c|}{} & $\mathrm{PD}+$ & $\mathrm{SM}$ \\
\hline $\mathrm{x}$ & 0.0017 & $3.8921 \mathrm{e}-08$ \\
\hline $\mathrm{y}$ & 0.000248 & $1.9359 \mathrm{e}-08$ \\
\hline $\mathrm{z}$ & 0.0031 & $2.9866 \mathrm{e}-04$ \\
\hline$\alpha \beta \gamma$ & 0.0162 & 0.0791 \\
\hline
\end{tabular}


Finally, the effort of each controller on the articular variables are obtained on table 2 . And it is observed that the control by SM requires a lower energy with respect to the PD + which makes it more efficient in terms of energy consumption.

Table 2. control effort

\begin{tabular}{|c|c|c|}
\cline { 2 - 3 } \multicolumn{1}{c|}{} & PD + & SM \\
\hline 1 & 0.0271 & 0.0038 \\
\hline 2 & 0.0210 & 0.0019 \\
\hline 3 & 0.1378 & 0.0494 \\
\hline 4 & 0.1033 & 0.0898 \\
\hline 5 & 0.0980 & 0.0836 \\
\hline 6 & 0.1373 & 0.0868 \\
\hline
\end{tabular}

\section{Conclusions}

It is possible to conclude that the non-linear controllers based on cancellation of the mathematical non linearities on the dynamical system (in this case the parallel mechanism HUNT-RUS) achieve excellent performance in terms of the error tracking the trajectories obtaining a very similar MSE of the error signals, nevertheless is very important to have a complete mathematical representation of the model to successfully cancel the nonlinear behaviours

In terms of the control signals the sliding modes control obtain a less torque, using less energy, but whit a high-speed overshoot caused by the attractive control that act saturating the actuators. In the PD+ controller is possible to conclude that uses more energy and higher torques than the sliding modes control technique, but avoiding the overshoots mentioned.

In terms of the implementation is important to mention that the sliding modes control is more difficult to implement.

\section{Acknowledgement}

The research for paper was supported by Nueva Granada Military University, through the project ING-IMP-2658.

\section{References}

1 Yin-Yen Kuo, Chiao-Hua Cheng and Shao-Kang Hung, "3D-Printed Linear Positioner with Micrometer Accuracy”, ICMME (2016)

2 Hermes Giberti, Luca Sbaglia and Marco Silvestri "Mechatronic Design for an Extrusion-Based Additive Manufacturing Machine", Machines, (2017)
Husein Zilullah Toresano, La Ode And Prajitno, Prawito., "Design of 3-Axis Cartesian Robot Manipulator" (2017)

4 I. Zabalza, J. Ros, J.J. Gil, J.M. Pintor, J.M. Jimenez, Tri-Scott. A new kinematic Structure for a 6-dof decoupled parallel manipulator, Workshop on fundamental issues and future research directions for parallelmechanisms and manipulators, pp.12-15, Québec, (2002).

5 E Mirshekari, A Ghanbarzabeh, K Heidari Shirazi, Structure Comparison and Optimal Design of 6RUS Parallel Manipulator Based on Kinematic and Dynamic Performances, LAJSS, Vol. 13, pp $2414-2438,2016$.

6 Vázquez H. Jesús, Cuenca J. Francisco, Cinemática Inversa y Análisis Jacobiano del Robot Paralelo Hexa, Memorias Somim, Vol. 1, No. 4, pp 800-810, Septiembre, (2009), Sonora. México.

7 A Zubizarreta, I Cabanes, E Portillo, D Orive, M Marcos Modelo dinamico de robots paralelos considerando sensorica redundante universidad del Pais Vasco 2006.

8 M Díaz., H Quintero, L A Mejía, G Holguin, MH errera, C Mesa, G Daraviña ., Aplicación de los robots paralelos", En Manipuladores paralelos: Sintesis, análisis y aplicaciones, Universidad Tecnológica de Pereira.

9 A. Arian, B. Danaei, M. Tale-Masouleh, Kinematic and Dynamic Analyses of Tripteron, an Over-Constrained 3-DOF Translational Parallel Manipulator, through Newton-Euler Approach" AUT Journal of Modeling and Simulation, (2018), pp 61-70

10 J. Ruiz-García, D. Chaparro-Altamirano, R. Zavala-Yoe and R. Ramírez-Mendoza, Direct and Inverse Dynamics Modeling of a 6-PUS Parallel Robot, ICMEAE, Morelos, (2013), pp. 21-26.

11 J. Uniyal, M. Joshi and P. K. Juneja, "Set point tracking capability and comparitive response of PI-PD controller with PI, PD and PID controller," ICACCA (Spring), Dehradun, (2016), pp. 1-6.

12 L. Jun, W. Xiaoguang, W. Yuqi and L. Qi, "Continuous terminal sliding mode control of a 6 DOF wire-driven parallel robot, ROBIO, Macau, (2017), pp. 1757-1762.

13 Z. Caihong and Z. Hongtao, Dynamic Sliding Mode Control Applied in Parallel Robot System, DCABES, Wuxi, (2011), pp. 405-408. 